\title{
The Application of Cloud computing in Hospital Information Platform
}

\author{
Maobao YANG \\ School of Information Science and Technology \\ Jiujiang University \\ Jiujiang,Jiangxi,China
}

\begin{abstract}
This paper studies three important application field of cloud computing in the construction of hospital information technology platform: Cloud data center, mobile medical desktop clouds and regional medical cloud. The article analyses the present situation, application effect, and the future development may face difficulties. Secondly, this article realization of the cloud data center design through the function definitions, requirements analysis and system architecture, mobile medical desktop clouds and regional medical cloud deployment, and focuses on the impact of the application of cloud data center and mobile medical desktop cloud on Hospital of traditional IT architecture and application effect.
\end{abstract}

Keywords-Mathematics; Cloud computing; Hospital Informatization; Infrastructure;

\section{INTRODUCTION}

With the development of medical service quality in hospital information construction, it bring a better and higher patient satisfaction, at the same time, the hospital also confronted with hitherto unknown challenge. The continuous expansion of the hospital scale, the number of outpatient, inpatient and business systems of increasing load demand, together with the construction of buildings, the function of the relocation and transformation will inevitably aggravate the hospital business system, storage security of medical data has also put forward higher requirements. A series of problems caused by the expansion of space server, rising energy and labor costs, etc., has been plagued by almost all hospitals. Hospital information system application of many kinds, including: HIS hospital information system, PACS system, LIS image inspection system, financial system, EMR electronic medical record system, paperless office business system OA system and decision support system etc. every kind of, covering financial, medical, scientific research, personnel, business process.

Therefore, hospital need a effectively improve the utilization rate of resources, cost control, business and strong ability to adapt, with high automation and intelligent management level of new technology and new development model to promote the development of hospital informatization, make the hospital IT management and maintenance work is simple and fast, take the initiative to provide service for the clinical departments.

\section{THE DESIGN OF SERVER ARCHITECTURE}

Figure 1 is the data center of the blade server architecture. First of all, the production room and disaster recovery room provide two server blades, based on virtual hardware resources to construct RAC cluster and HIS system, not only access to a powerful computing capability, but also to ensure business continuity, in which a single server or the entire data center server downtime situation, another data center server automatically take over the service, to ensure the normal operation of business. Secondly, the production room left in the six sets of blade server virtualization, build server cluster function big, the virtual pool of resources are abstracted into all hardware resources, to a virtual multi pattern, create multiple virtual server in a cluster, as the other business systems (such as PACS systems, anesthesia and material management system) to provide services. In addition, in order to ensure high availability of core business system, built a disaster recovery data center, using the backup mechanism, the disaster recovery room within the two server blades as a hot standby server, server downtime in the production room all the circumstances, can completely take over (to ensure sufficient memory space case) all business production server room operation in addition, the; when the deployment of mobile medical desktop cloud, in order to prevent the peak period for managed desktop simultaneously a large number of access, resulting in the same network segment large flow network impact, affecting the performance of the production environment system, therefore establish separate virtual desktop server cluster using disaster recovery room in another segment of the four server blades to ensure stable operation of the business. Finally, according to the plan, and gradually put the key application system dispersed in the original frame on the server gradually migrated to the blade server cluster and manage. And according to the original frame server, can be re unified planning and deployment of its virtual processing after each physical server to make the abstract logical resource pool, to create multiple virtual machine server, use for the development and testing by the new system and new business. 


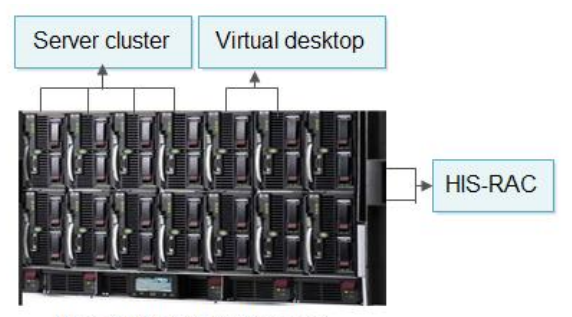

The production data center

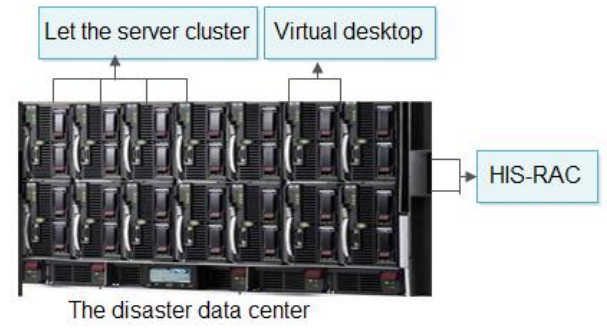

Fig.1 architecture of blade server in data center

\section{NETWORK ARCHITECTURE DESIGN}

According to characteristics of hospital data center network and demand, the paper puts forward the technology method that is as shown in figure 2 .

(1) Gigabit backbone network and the big two layer technology. In order to guarantee high speed and stable operation of hospital business system, provide high bandwidth network as the support, to meet such as remote expert consultation, medical imaging and inspection, medical research and teaching applications such as information transmission needs, improve the satisfaction and the service quality of the hospital medical workers. The establishment of Gigabit backbone network and Gigabit to the desktop terminal of the Ethernet, the hospital application rescued from past low-speed links, providing a new high-speed operation platform. In addition, in order to meet the demand of massive data forwarding southeast hospital, cloud data center using two layers of flat network architecture, effectively improve the data forwarding capacity and treatment efficiency.

(2) Network cache deployment. Virtualization technology is the foundation to realize the cloud computing platform, although through the server virtualization mode can greatly improve the server performance and storage throughput, but because of the virtual machine is a superposition of multiple physical servers, so need more traditional environment several times or even dozens of times network traffic and performance requirements. At the same time, more concentrated in highly intensive application environment, on the basis of the reliability of the network requirements are more demanding, fault local network, the effect on the business to be more serious than the traditional environment. Therefore, the deployment of network data center, S12500, S7500E series switches were used in the core and access position, which provides high volume port cache to deal with $200 \mathrm{~ms}$ bursty traffic, can effectively reduce the probability for bursty traffic caused by network fault, meet the demand of traffic impact.

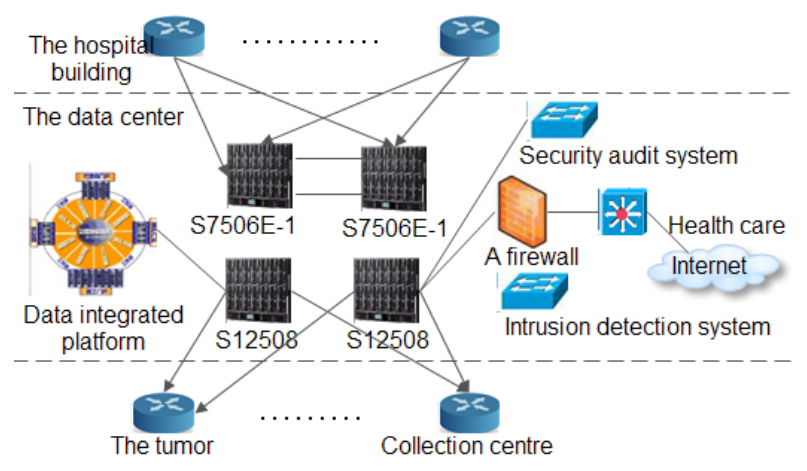

Fig.2 The topology structure of hospital network

\section{THE DESIGN OF STORAGE SERVER}

Storage is as the core of hospital information system, how to break through the limited capacity and performance bottlenecks, to respond to the hospital very large scale data volume and storage capacity doubled elasticity of demand resulting from the test, is a major challenge for cloud data center storage. The hospital has a HIS platform and PACS storage architecture as shown in Figure 3 and Figure 4, the HIS system runs on a two set of rack server, and constructs a Oracle R2 RAC real-time cluster environment, using automatic storage management (ASM) disk management mode, all data storage to the HP EVA4400 storage system, two sets of the host through the fiber channel (FC) two controllers are respectively connected with the direct connection of EVA4400 redundant, and has two sets of database level backup DataGuard physical backup database host, respectively by means of real-time and non-real time method and the production base for realization of $\log$ synchronization; PACS system running on a single physical server of its production system frame, data storage in the CX300 storage devices, the archive data stored in the MSA200 storage device.

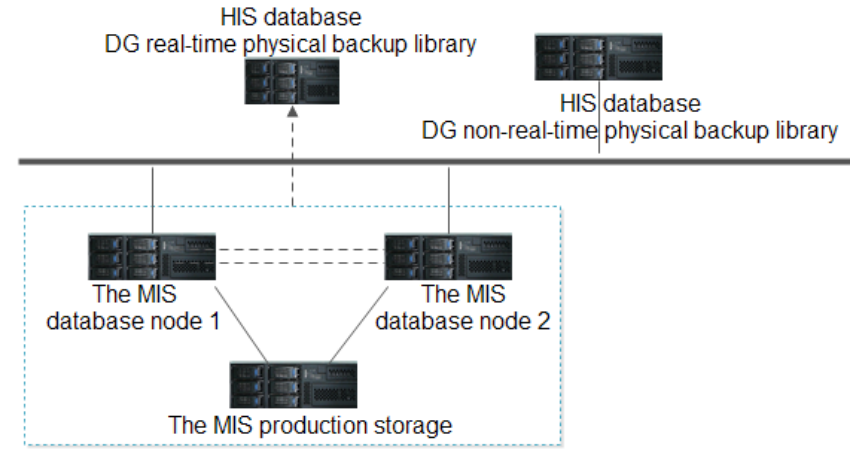

Fig. 3 topological structure of HIS core database system

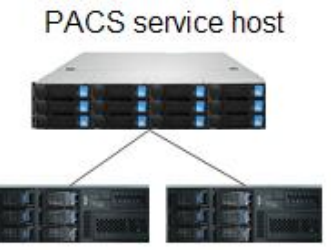

Fig.4 topological structure of PACS system 


\section{IMPLEMENTATION PLAN OF CLOUD DESKTOP}

In order to improve the traditional status of hospital PC desktop terminal system with independent scattered, difficult management, select VMware View desktop virtualization solution to realize system usability and optimization management, provide flexible free desktop environment, reduces the cost of investment in the project, the use of four independent HP blade server and VMware software tools to build to solve the desktop virtualization based on blade server scheme. In the VMware ESXi and VMware View desktop virtualization tools to construct a blade server cluster with, the establishment of a unified resource pool, the virtual machine to create large quantities of one to many service mode, and each virtual machine is a complete virtual desktop, with operating system, applications and user individuation configuration, since all data to maintain in the corporate firewall, encryption provides a built-in SSL from nonmanaged device to the safety insurance channel virtual desktop, so VMware View can reduce the data leakage and loss risk. Through the VMware vCenter management tools, management staff to focus on monitoring and management of all virtual machines in a resource pool. Using the View Client tool, the user can from the traditional PC, Thin Client or other mobile devices (iPad, intelligent mobile phone etc.) access to the desktop environment.

\section{THE APPLICATION EFFECT OF CLOUD COMPUTING IN THE HOSPITAL INFORMATION PLATFORM}

The effect of cloud data center application is as shown in figure 5. After the hospital cloud computing platform is completed, the application of primordium in physical server HIS, PACS, financial system in turn smoothly online migrated to the new cloud platform. Technology innovation by using cloud computing, realizes the resource integration management and distribution according to need, the application of fast deployment, demand response time, system portability enhancement, mobile medical and office, network performance is stable and reliable, reduce cost, low carbon energy saving target. One of the most important feature is: the use of high availability to eliminate single points of failure; through the promotion of resources to avoid waste utilization; through the centralized storage to ensure data security; operating system and hardware decouple hardware implementation of transverse flexible extension; application level disaster recovery system and ensure continuous data security.

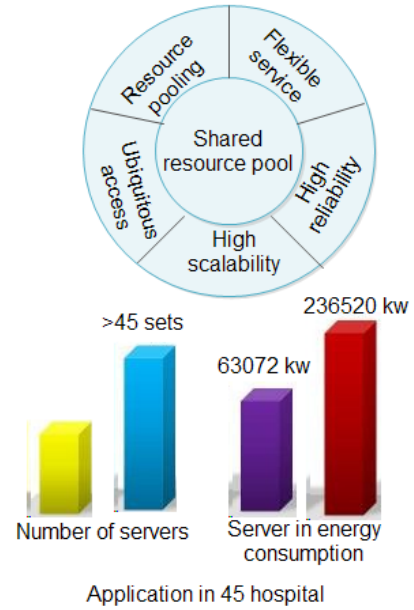

Fig.5 The application effect of cloud data center

\section{CONCLUSIONS}

After in-depth study cloud computing and the development status of hospital informationization foundation theory, the paper developed a set of digital hospital informationization conforms to the current development construction scheme, used to make up the insufficiency of traditional mode for hospital information construction, after the completion of requirements analysis and architecture analysis of data center construction based on Cloud Computing, combined with the needs of the project, carried out a cloud data center construction and medical desktop cloud application practice, fully demonstrated the cloud computing a good effect has been achieved in the construction of hospital information. This article in the elaboration process, pay attention to the combination of theory with practice, will be fully digital hospital information construction in close combination of cloud computing theory and technology characteristics, thereby significantly enhance the level of development of hospital informatization.

\section{REFERENCES}

[1] Wu Zhuhua. The analysis of the core technology of cloud computing [M]. Beijing: People's Posts and Telecommunications Press, 2011.2.

[2] ZTE University. Dialogue of cloud computing [M]. Beijing: People's Posts and Telecommunications Press, 2012.8.

[3] QingKe research center. China cloud computing industry Investment Research Report In 2013 [R/0L]. [2013-7-30].

[4] Zhang Jie. Cloud computing - hospital information system and the opportunities and challenges $[\mathrm{J}]$. China medical education technology, 2012, (5): 260-562.

[5] Ma Xikun, in Jingjie, Yang Guobin. Effect of storage virtualization technology in the platform of hospital information system $[\mathrm{J}]$. China Medical equipment, 2011, $26 \quad$ (10): $39-40, \quad 50$ 